PROCEEDINGS OF THE

AMERICAN MATHEMATICAL SOCIETY

Volume 138, Number 3, March 2010, Pages 871-879

S 0002-9939(09)10156-9

Article electronically published on October 21, 2009

\title{
SOME REMARKS ON BOUNDED EARTHQUAKES
}

\author{
DRAGOMIR ŠARIĆ
}

(Communicated by Mario Bonk)

\begin{abstract}
We show that an earthquake of a geometrically infinite hyperbolic surface induces an asymptotically conformal change in the hyperbolic metric if and only if the measured lamination associated with the earthquake is asymptotically trivial on the surface. Then we show that the contraction along earthquake paths is continuous in the Teichmüller space of any hyperbolic surface. Finally, we show that if a measured lamination vanishes while approaching infinity at a rate higher than the distance to the boundary, then it must be trivial.
\end{abstract}

\section{INTRODUCTION}

An earthquake $E$ of a hyperbolic surface $M$ is obtained by partitioning $M$ into the strata and by moving one stratum at a time. The support of an earthquake $E$ is a geodesic lamination $\lambda$ on $M$. Each stratum of $E$ is either a geodesic from $\lambda$ or a component of the complement of $\lambda$ in $M$. The earthquake $E$ does not change the shape of each stratum (i.e. $E$ is a hyperbolic isometry on each stratum) and it moves each stratum to the left relative to any other stratum. For all the details, see Thurston [11] or Section 2.

The relative movement to the left between any two strata of $E$ is best described by a transverse measure to $\lambda$ which is invariant under homotopies relative to $\lambda$. A geodesic lamination together with a transverse measure is called a measured lamination. An earthquake $E$ is uniquely determined (up to post-composition with a hyperbolic isometry) by its measured lamination. Any earthquake $E$ of $M$ onto another hyperbolic surface $M_{1}$ induces a homeomorphism between the boundaries of their corresponding universal coverings (we take the upper half-plane $\mathbf{H}$ as universal coverings); i.e. $E$ induces a homeomorphism of the extended real line $\hat{\mathbf{R}}=\mathbf{R} \cup\{\infty\}$ which conjugates the uniformizing Fuchsian group of $M$ onto the uniformizing Fuchsian group of $M_{1}$. Thurston [11] showed that any homeomorphism of $\hat{\mathbf{R}}$ is induced by an earthquake $E$ of the upper half-plane $\mathbf{H}$, and if a homeomorphism conjugates a Fuchsian group $G$ onto another Fuchsian group, then $E$ is an earthquake of $\mathbf{H} / G$.

An earthquake $E$ of the upper half-plane $\mathbf{H}$ induces a quasisymmetric homeomorphism of $\hat{\mathbf{R}}$ if and only if the transverse measure of $E$ is uniformly bounded on all hyperbolic arcs of length 1 (see 9, [5, 7, 4, 10] and 11, or Section 2). Moreover, an earthquake $E$ of $\mathbf{H}$ induces a symmetric homeomorphism of $\hat{\mathbf{R}}$ if and

Received by the editors September 10, 2008.

2010 Mathematics Subject Classification. Primary 30F60; Secondary 32G15.

This work was in part supported by PSC CUNY grant PSC-REG-39-386.

(C)2009 American Mathematical Society Reverts to public domain 28 years from publication 
only if the transverse measure of $E$ tends to zero uniformly on hyperbolic arcs of length 1 which converge to the boundary $\hat{\mathbf{R}}$ of $\mathbf{H}$ (see [5, [7] and [10, or Section 2).

A symmetric homeomorphism of $\hat{\mathbf{R}}$ extends to an asymptotically conformal quasiconformal map of $\mathbf{H}$, and an asymptotically conformal quasiconformal map of $\mathbf{H}$ extends to a symmetric homeomorphism of $\hat{\mathbf{R}}$ (see [6], 3] or Section 2). A proper generalization of the space of all symmetric homeomorphisms of $\hat{\mathbf{R}}$ for a geometrically infinite hyperbolic surface $M$ is the space of all asymptotically conformal quasiconformal maps from $M$ onto variable hyperbolic surfaces $M_{1}$ up to bounded homotopy (see [6], 2]). A lift to $\mathbf{H}$ of an asymptotically conformal quasiconformal map of $M$ does not induce a symmetric homeomorphism of $\hat{\mathbf{R}}$ (unless $M=\mathbf{H}$ ). Our first result is a characterization of earthquakes of $M$ whose lifts to $\mathbf{H}$ agree on the boundary $\hat{\mathbf{R}}$ with the continuous extensions of lifts of asymptotically conformal quasiconformal maps of $M$ (see Theorem 3.1).

Let $M$ be a geometrically infinite hyperbolic surface and let $E$ be an earthquake of $M$. Let $\tilde{E}: \mathbf{H} \rightarrow \mathbf{H}$ be a lift of $E$ to the universal covering and let $\left.\tilde{E}\right|_{\hat{\mathbf{R}}}$ be its continuous extension to the boundary $\hat{\mathbf{R}}$. Let $f: M \rightarrow M_{1}$ be a quasiconformal map. Denote by $\tilde{f}: \mathbf{H} \rightarrow \mathbf{H}$ a lift of $f$ to the universal covering $\mathbf{H}$ and by $\left.\tilde{f}\right|_{\hat{\mathbf{R}}}$ the continuous extension to $\hat{\mathbf{R}}$. The measured lamination associated with $E$ is said to be asymptotically trivial on $M$ if the supremum of the measures of hyperbolic arcs of length 1 outside a compact subset $K$ of $M$ is approaching zero as $K$ exhausts $M$.

Theorem 1. An earthquake $E$ of a geometrically infinite hyperbolic surface $M$ induces a homeomorphism $\left.\tilde{E}\right|_{\hat{\mathbf{R}}}$ of $\hat{\mathbf{R}}$ which agrees with an induced homeomorphism $\left.\tilde{f}\right|_{\hat{\mathbf{R}}}$ of an asymptotically conformal quasiconformal map $f: M \rightarrow M_{1}$ if and only if the transverse measure of $E$ is asymptotically trivial on $M$.

Let $M L_{b}(\mathbf{H})$ denote the space of all bounded measured laminations on $\mathbf{H}$. Each quasisymmetric homeomorphism $h: \hat{\mathbf{R}} \rightarrow \hat{\mathbf{R}}$ is obtained by continuously extending to $\hat{\mathbf{R}}$ an earthquake $E$ of $\mathbf{H}$ with associated bounded measured lamination. This gives an earthquake measure map $\mathcal{E M}: T(\mathbf{H}) \rightarrow M L_{b}(\mathbf{H})$, where $T(\mathbf{H})$ is the universal Teichmüller space; namely, $T(\mathbf{H})$ is the space of all quasisymmetric maps of $\hat{\mathbf{R}}$ modulo post-composition with $P S L_{2}(\mathbf{R})$. If $\mathbf{H}$ is replaced by a compact hyperbolic surface $S$, then the weak* topology on $M L(S)$ makes the earthquake measure map $\mathcal{E} \mathcal{M}: T(S) \rightarrow M L(S)$ a homeomorphism (see [8]). The question concerning a natural topology on $M L_{b}(\mathbf{H})$ which makes $\mathcal{E} \mathcal{M}: T(\mathbf{H}) \rightarrow M L_{b}(\mathbf{H})$ into a homeomorphism is still open (by a natural topology on $M L_{b}(\mathbf{H})$ we mean a topology given in terms of measured laminations on $\mathbf{H}$ without reference to the earthquake measure map). However, we show that the earthquake measure map is natural for the multiplication of the measured laminations by a positive parameter (see Theorem 4.1).

Theorem 2. The scaling measure map $H:[0,1] \times T(\mathbf{H}) \rightarrow T(\mathbf{H})$ given by

$$
H(t,[h])=\left[\left.E^{(1-t) \mathcal{E M}([h])}\right|_{\hat{\mathbf{R}}}\right]
$$

is continuous.

The above theorem is true for Teichmüller spaces of an arbitrary hyperbolic surface $M$ as well. A direct corollary to the above theorem is that the Teichmüller 
space $T(M)$ is contractible (also the closed subspace of $T(M)$ of classes with asymptotically conformal representatives is contractible). This was originally proved by Douady and Earle [1. We obtained a new proof of this fact using earthquakes (see Corollary 4.1).

Finally, we consider measured laminations whose measures vanish as they approach the boundary. Gardiner-Hu-Lakic [5] and Hu [7] considered earthquakes whose measured laminations $\mu$ satisfy $\mu=o\left(\delta^{\alpha}\right)$, for $\alpha>0$. (By the definition, $\mu=o\left(\delta^{\alpha}\right)$ if the supremum of $\mu(I)$ over all hyperbolic arcs of length 1 which are at a distance at most $\delta$ from the boundary is $o\left(\delta^{\alpha}\right)$.) We show that if $\alpha=1$, i.e. $\mu=o(\delta)$, then $\mu$ is a trivial measured lamination, i.e. $\mu=0$ (see Proposition 5.1).

\section{Preliminaries: Bounded earthquakes}

Let $\mathbf{H}$ be the upper half-plane with the metric $\rho(z)=\frac{|d z|}{y}$. Denote by $\hat{\mathbf{R}}=$ $\mathbf{R} \cup\{\infty\}$ the boundary at infinity of $\mathbf{H}$. We identify the hyperbolic plane with the model $(\mathbf{H}, \rho)$.

Let $M$ be a Riemann surface of infinite geometric type. The universal covering of $M$ is the hyperbolic plane $\mathbf{H}$. The Riemann surface $M$ supports a unique hyperbolic metric in its conformal class which is the projection of $\rho$ under the universal covering by $\mathbf{H}$. Let $G$ be a subgroup of $P S L_{2}(\mathbf{R})$ such that $M$ is conformal and isometric to $\mathbf{H} / G$. From now on, we fix one such $G$ and the identification $M=\mathbf{H} / G$.

Definition 2.1. A geodesic lamination $\lambda$ on $\mathbf{H}$ is a closed subset $|\lambda|$ of $\mathbf{H}$ together with an assignment of a foliation of $|\lambda|$ by geodesics of $\mathbf{H}$. A geodesic lamination $\lambda_{G}$ on $M=\mathbf{H} / G$ is a closed subset $\left|\lambda_{G}\right|$ of $\mathbf{H}$ which is foliated by geodesics of $\mathbf{H}$ such that the subset $\left|\lambda_{G}\right|$ and the foliation of $\left|\lambda_{G}\right|$ are invariant under $G$. A stratum of $\lambda$ is either a geodesic of the foliation of $|\lambda|$ or a connected component of $\mathbf{H} \backslash|\lambda|$.

Remark. A component of $\mathbf{H} \backslash|\lambda|$ is an open hyperbolic polygon whose boundary in $\mathbf{H}$ consists of geodesics of the foliation. The polygon is possibly infinite-sided and can contain open intervals of $\hat{\mathbf{R}}$ on its boundary at infinity.

Remark. For simplicity of notation, a geodesic lamination on $\mathbf{H}$ which is invariant under the Fuchsian group $G$ is said to be a geodesic lamination on $\mathbf{H} / G$.

Definition 2.2. Let $\lambda$ be a geodesic lamination on $\mathbf{H}$. A measured lamination $\mu$ on $\mathbf{H}$ with support $\lambda$ is an assignment of a positive, Radon measure to each closed, finite hyperbolic arc $I$ in $\mathbf{H}$ whose support is $I \cap|\lambda|$ and which is invariant under homotopies which preserve the foliation of $|\lambda|$. (We do not require that the homotopies preserve the endpoints of $I$.) A measured lamination on $\mathbf{H} / G$ is an assignment of a measured lamination $\mu_{G}$ on $\mathbf{H}$ such that the support $\lambda_{G}$ is a measured lamination on $\mathbf{H} / G$ (i.e. $\lambda_{G}$ is invariant under $G$ ) and that the measure $\mu_{G}$ is invariant under the action of $G$.

Remark. For simplicity of notation, a measured lamination on $\mathbf{H}$ which is invariant under the Fuchsian group $G$ is said to be a measured lamination on $\mathbf{H} / G$.

We define an earthquake of the hyperbolic plane $\mathbf{H}$ following Thurston [11.

Definition 2.3. An earthquake $E$ of $\mathbf{H}$ whose support is a geodesic lamination $\lambda$ is a bijective map $E: \mathbf{H} \rightarrow \mathbf{H}$ such that the restriction of $E$ to any stratum of $\lambda$ is in $P S L_{2}(\mathbf{R})$ and such that $E$ moves strata of $\lambda$ to the left relative to each other. Namely, for any stratum $g$ of $\lambda$ we have that $\left.E\right|_{g} \in P S L_{2}(\mathbf{R})$, and for any two 
strata $g_{1}, g_{2}$ of $\lambda$ we have that $\left.\left(\left.E\right|_{g_{1}}\right)^{-1} \circ E\right|_{g_{2}}$ is a hyperbolic isometry whose axis is weakly separating the two strata $g_{1}$ and $g_{2}$, and which translates $g_{2}$ to the left as seen from $g_{1}$. An earthquake $E$ of $\mathbf{H}$ is said to be an earthquake of $\mathbf{H} / G$ if the support $\lambda$ is invariant under $G$, and for every $\gamma \in G$ there exists $\gamma_{E} \in P S L_{2}(\mathbf{R})$ such that $E \circ \gamma=\gamma_{E} \circ E$.

An earthquake $E$ of $\mathbf{H}$ naturally induces a measured lamination $\mu$ with support $\lambda$ as follows. The measure $\mu$ on each compact hyperbolic $\operatorname{arc} I$ is approximated by the sum of the translation lengths between the comparison isometries of $n$ consecutive strata of the support $\lambda$ of $E$ which intersect $I$ such that the distance between any two consecutive strata is small for $n$ large (see [11, [5]). The measure $\mu(I)$ is obtained by taking the limit of the sum of translation lengths as $n \rightarrow \infty$. It is clear that $\mu$ is homotopy invariant. An earthquake $E$ is uniquely determined by its measured lamination up to post-composition with $P S L_{2}(\mathbf{R})$ [11. A measured lamination corresponding to an earthquake is called an earthquake measure. Moreover, $E$ is an earthquake on $\mathbf{H} / G$ if and only if the earthquake measure of $E$ is a measured lamination on $\mathbf{H} / G$.

An earthquake of $\mathbf{H}$ is not necessarily a homeomorphism of $\mathbf{H}$. A discontinuity of an earthquake appears at the support geodesics of the earthquake measure $\mu$ which are atoms of $\mu$. However, an earthquake always extends by continuity to a homeomorphism of the boundary $\hat{\mathbf{R}}$ of $\mathbf{H}$. Thurston 11 proved that any homeomorphism of $\hat{\mathbf{R}}$ can be obtained as the continuous extension of an earthquake of $\mathbf{H}$ to the boundary $\hat{\mathbf{R}}$. In addition, any homeomorphic deformation of the hyperbolic structure on $M=\mathbf{H} / G$ is obtained by an earthquake of $M$ [11.

Definition 2.4. An earthquake measure $\mu$ is said to be bounded if

$$
\|\mu\|=\sup _{I} \mu(I)<\infty,
$$

where the supremum is over all length 1 closed hyperbolic arcs $I$ in $\mathbf{H}$.

An earthquake $E$ of $\mathbf{H}$ extends by continuity to a quasisymmetric map of $\hat{\mathbf{R}}$ if and only if the earthquake measure $\mu$ of $E$ is bounded (see [9], 5], 11], 44, 10]).

Definition 2.5. An earthquake measure $\mu$ is said to be asymptotically trivial if for every $\epsilon>0$ there exists a compact subset $K \subset \mathbf{H}$ such that

$$
\sup _{I \cap K=\emptyset} \mu(I)<\epsilon
$$

where the supremum is over all length 1 hyperbolic arcs which do not intersect $K$.

A quasisymmetric homeomorphism of $\hat{\mathbf{R}}$ is said to be symmetric if it distorts symmetric triples into almost symmetric triples on small scales [ 6 . Equivalently, a quasisymmetric homeomorphism $h: \hat{\mathbf{R}} \rightarrow \hat{\mathbf{R}}$ is symmetric if and only if there exists a quasiconformal extension $f: \mathbf{H} \rightarrow \mathbf{H}$ of $h$ such that for every $\epsilon>0$ there exists a compact set $K \subset \mathbf{H}$ with $\left\|\left.\operatorname{Belt}(f)\right|_{\mathbf{H} \backslash K}\right\|_{\infty}<\epsilon$, where $\operatorname{Belt}(f)=\bar{\partial} f / \partial f$ is the Beltrami coefficient of $f$.

It was proved by Gardiner, Hu and Lakic 5 that an earthquake $E$ extends to a symmetric homeomorphism of $\hat{\mathbf{R}}$ if and only if the earthquake measure $\mu$ of $E$ is asymptotically trivial (see also [7] and [10] for different proofs). 


\section{Asymptotically CONFormal structures on INFInite SURFACES VIA EARTHQUAKES}

Let $M=\mathbf{H} / G$ be a geometrically infinite hyperbolic surface. A quasiconformal map $f: M \rightarrow M_{1}$ is said to be asymptotically conformal on $M=\mathbf{H} / G$ if for any $\epsilon>0$ there exists a compact set $K \subset M$ such that $\left\|\left.\operatorname{Belt}(f)\right|_{M \backslash K}\right\|_{\infty}<\epsilon$, where $\operatorname{Belt}(f)=\bar{\partial} f / \partial f$ is the Beltrami coefficient of $f$.

Definition 3.1. An earthquake measure $\mu_{G}$ on $\mathbf{H} / G$ is said to be asymptotically trivial on $\mathbf{H} / G$ if for any $\epsilon>0$ there exists a compact subset $K$ of $\mathbf{H} / G$ such that $\sup _{I \cap \tilde{K}=\emptyset} \mu_{G}(I)<\epsilon$, where the supremum is over all closed hyperbolic arcs $I$ of length 1 which do not intersect the lift $\tilde{K}$ of $K$ to $\mathbf{H}$.

Theorem 3.1. Let $f: M \rightarrow M_{1}$ be a quasiconformal map between Riemann surfaces $M=\mathbf{H} / G$ and $M_{1}=\mathbf{H} / G_{1}$, where $G, G_{1}<P S L_{2}(\mathbf{R})$. Fix a lift $\tilde{f}: \mathbf{H} \rightarrow \mathbf{H}$ of $f$ which conjugates $G$ onto $G_{1}$ and denote by $h: \hat{\mathbf{R}} \rightarrow \hat{\mathbf{R}}$ the continuous extension of $\tilde{f}$ to $\hat{\mathbf{R}}$. Let $E: \mathbf{H} \rightarrow \mathbf{H}$ be the earthquake on $\mathbf{H} / G$ whose continuous extension to $\hat{\mathbf{R}}$ equals $h$. Then the earthquake measure $\mu_{h}$ of $E$ is asymptotically trivial on $\mathbf{H} / G$ if and only if $f$ is homotopic to an asymptotically conformal map on $\mathbf{H} / G$ through a bounded homotopy.

Proof. Let $f: M \rightarrow M_{1}$ be homotopic to an asymptotically conformal map through a bounded homotopy and let $h: \hat{\mathbf{R}} \rightarrow \hat{\mathbf{R}}$ be a continuous extension to $\hat{\mathbf{R}}$ of a lift $\tilde{f}: \mathbf{H} \rightarrow \mathbf{H}$ of $f$. Let $F$ be the Douady-Earle extension of the quasisymmetric map $h: \hat{\mathbf{R}} \rightarrow \hat{\mathbf{R}}$ to $\mathbf{H}$ (see [1]). Then $F$ induces an asymptotically conformal map on $\mathbf{H} / G$ (see [3]); namely, for every $\epsilon>0$ there exists a compact set $K \subset \mathbf{H} / G$ such that $\left\|\left.\operatorname{Belt}(F)\right|_{\mathbf{H} \backslash \tilde{K}}\right\|_{\infty}<\epsilon$, where $\tilde{K}$ is the lift of $K$ to $\mathbf{H}$ and $\operatorname{Belt}(F)=\frac{\bar{\partial} F}{\partial F}$. Assume on the contrary that the earthquake measure $\mu_{h}$ of $E$ is not asymptotically trivial on $M=\mathbf{H} / G$. Namely, there exists $\epsilon_{0}>0$ and a sequence $I_{n} \subset \mathbf{H}$ of hyperbolic arcs of length 1 such that $\mu\left(I_{n}\right) \geq \epsilon_{0}>0$ and the preimage in $\mathbf{H}$ of any compact subset of $\mathbf{H} / G$ intersects only finitely many $I_{n}$ 's.

Let $i \in \mathbf{H}$ be an imaginary unit and let $\gamma_{n} \in P S L_{2}(\mathbf{R})$ be such that $\gamma_{n}\left(I_{n}\right) \ni i$. Let $\mu_{n}=\left(\gamma_{n}\right)_{*}\left(\mu_{h}\right)$ be the push-forward of $\mu_{h}$ by $\gamma_{n}$. Note that the support of $\mu_{n}$ is $\gamma_{n}\left(\lambda_{h}\right)$, where $\lambda_{h}$ is the support of $\mu_{h}$. Then $\mu_{n}$ is the earthquake measure of an earthquake $E^{\mu_{n}}$ which agrees with $h \circ \gamma_{n}^{-1}$ on $\hat{\mathbf{R}}$ up to post-composition with an element of $P S L_{2}(\mathbf{R})$. Since $\left\|\mu_{n}\right\|=\left\|\mu_{h}\right\|$ for each $n$, it follows that there is a subsequence $\mu_{n_{k}}$ of the sequence $\mu_{n}$ which converges in the weak* sense to a measured lamination $\mu_{*}$ with $\left\|\mu_{*}\right\| \leq\left\|\mu_{h}\right\|$. Note that $\mu_{*}$ is a non-zero earthquake measure because $\mu_{h}\left(I_{n}\right)=\mu_{n}\left(\gamma_{n}\left(I_{n}\right) \geq \epsilon_{0}\right.$. By abuse of notation we write $\mu_{n}$ in place of $\mu_{n_{k}}$.

The restriction $\left.E^{\mu_{n}}\right|_{\hat{\mathbf{R}}}$ to $\hat{\mathbf{R}}$ of a properly normalized sequence of earthquakes $E^{\mu_{n}}$ converges pointwise to the restriction $\left.E^{\mu_{*}}\right|_{\hat{\mathbf{R}}}$ of a properly normalized earthquake $E^{\mu_{*}}$ (see [10, Proposition 3.3]). Let $\delta_{n} \in P S L_{2}(\mathbf{R})$ be such that $\delta_{n} \circ h \circ$ $\left.\gamma_{n}^{-1}\right|_{\hat{\mathbf{R}}}=\left.E^{\mu_{n}}\right|_{\hat{\mathbf{R}}}$ for all $n$. Let $F_{n}$ be the Douady-Earle extension of $\delta_{n} \circ h \circ \gamma_{n}^{-1}$. Since the $I_{n}$ 's leave the preimage in $\mathbf{H}$ of every compact subset of $\mathbf{H} / G$ and by the conformal naturality of the Douady-Earle extension, we have that $\operatorname{Belt}\left(F_{n}\right)$ converges to zero uniformly on compact subsets of $\mathbf{H}$ because $F$ is asymptotically conformal by our assumption. In addition, since $\delta_{n} \circ h \circ \gamma_{n}^{-1}$ pointwise converges, it follows that $F_{n}$ converges to a Möbius map by the continuity properties of the Douady-Earle extension (see [1] and [3]). On the other hand, $\delta_{n} \circ h \circ \gamma_{n}^{-1}=\left.E^{\mu_{n}}\right|_{\hat{\mathbf{R}}}$ 
weakly converges to $\left.E^{\mu_{*}}\right|_{\hat{\mathbf{R}}}$ with $\mu_{*}$ non-trivial. This implies that the DouadyEarle extension of $\left.E^{\mu_{*}}\right|_{\hat{\mathbf{R}}}$ is not Möbius, which is a contradiction with continuity properties of the Doaudy-Earle extension.

Let $\mu_{h}$ be an asymptotically trivial earthquake measure on $\mathbf{H} / G$ corresponding to $h$. We need to show that the Douady-Earle extension $F$ of $h$ is asymptotically conformal. Assume on the contrary that there exists a sequence $z_{n} \in \mathbf{H}$ which leaves the preimage in $\mathbf{H}$ of every compact subset of $\mathbf{H} / G$ such that $\left|\operatorname{Belt}(F)\left(z_{n}\right)\right| \geq \epsilon_{0}$, for fixed $\epsilon_{0}>0$. Let $\gamma_{n} \in P S L_{2}(\mathbf{R})$ be such that $\gamma_{n}\left(z_{n}\right)=i \in \mathbf{H}$. Let $\mu_{n}=\left(\gamma_{n}\right)_{*} \mu_{h}$. Then there exists a subsequence $\mu_{n_{k}}$ of $\mu_{n}$ which converges in the weak* sense to a measured lamination $\mu_{*}$ with $\left\|\mu_{*}\right\| \leq\left\|\mu_{h}\right\|$. Without loss of generality we assume that the sequence $\mu_{n}$ converges. By the choice of $\gamma_{n}$, it follows that $\mu_{*}$ is the trivial measured lamination, i.e. $\mu_{*}=0$. This implies that there exists $\delta_{n} \in P S L_{2}(\mathbf{R})$ such that $\delta_{n} \circ h \circ \gamma_{n}^{-1}=\left.E^{\mu_{n}}\right|_{\hat{\mathbf{R}}}$ converges weakly to the identity, where $\left.E^{\mu_{n}}\right|_{\hat{\mathbf{R}}}$ is a properly normalized earthquake [10]. Let $F_{n}$ be the Douady-Earle extension of $\delta_{n} \circ h \circ \gamma_{n}^{-1}$. Then $\operatorname{Belt}\left(F_{n}\right)$ converges uniformly to 0 on compact subsets of $\mathbf{H}$. However, $\left|\operatorname{Belt}\left(F_{n}\right)(i)\right|=\left|\operatorname{Belt}(F)\left(z_{n}\right)\right| \geq \epsilon_{0}>0$, which is a contradiction.

\section{The EarthquaKe MeAsure map And THE CONTRACTIBILITy}

Given a quasisymmetric map $h: \hat{\mathbf{R}} \rightarrow \hat{\mathbf{R}}$, there is a unique earthquake $E_{h}$ of $\mathbf{H}$ whose continuous extension to $\hat{\mathbf{R}}$ coincides with $h$. Let $\mu_{h}$ be the earthquake measure of $E_{h}$ ( $\mu_{h}$ is bounded [10]). Thus we obtain a one-to-one assignment $[h]=P S L_{2}(\mathbf{R}) \circ h \mapsto \mu_{h}$ from the universal Teichmüller space $T(\mathbf{H})$ onto the space of bounded measured laminations $M L_{b}(\mathbf{H})$ of $\mathbf{H}$. (Note that $[h]$ denotes the class $P S L_{2}(\mathbf{R}) \circ h$ of a quasisymmetric map $h$ and that $T(\mathbf{H})$ is the space of all such classes of quasisymmetric maps.) We denote this map by $\mathcal{E M}: T(\mathbf{H}) \rightarrow M L_{b}(\mathbf{H})$ and call it the earthquake measure map. The earthquake measure map restricts to a map from the Teichmüller space of a hyperbolic Riemann surface $M$ onto the space of bounded measured laminations on $M$ (see [11).

If $M$ is a compact surface of genus at least two, then the weak* topology on $M L_{b}(M)$ makes the earthquake measure map $\mathcal{E} \mathcal{M}: T(M) \rightarrow M L_{b}(M)$ a homeomorphism (see [8]). For a geometrically infinite Riemann surface $M$ (including the possibility that $M=\mathbf{H}$ ), it is an open problem to find a natural topology on $M L_{b}(M)$ which would make $\mathcal{E} \mathcal{M}: T(M) \rightarrow M L_{b}(M)$ a homeomorphism. (One can induce a topology on $M L_{b}(M)$ by the pull-back of the topology on $T(M)$, but there is no information about such an assigned topology on $M L_{b}(M)$. A "natural" topology on $M L_{b}(M)$ should be given in terms of the measured laminations on the surface $M$ without the use of the quasisymmetric maps.) However, we show below that the earthquake measure map $\mathcal{E} \mathcal{M}: T(M) \rightarrow M L_{b}(M)$ is natural for the scaling of the earthquake measure.

Theorem 4.1. The scaling measure map $H:[0,1] \times T(\mathbf{H}) \rightarrow T(\mathbf{H})$ given by

$$
H(t,[h])=\left[\left.E^{(1-t) \mathcal{E M}([h])}\right|_{\hat{\mathbf{R}}}\right]
$$

is continuous.

Remark. The map $H:[0,1] \times T(\mathbf{H}) \rightarrow T(\mathbf{H})$ restricts to a continuous map $H$ : $[0,1] \times T(\mathbf{H} / G) \rightarrow T(\mathbf{H} / G)$, for any Fuchsian group $G$. 
Proof. Let $[h] \in T(\mathbf{H})$. We show that $H$ is continuous at $(t,[h])$. Let $\left[h_{n}\right] \in T(\mathbf{H})$ be a sequence converging to $[h]$ in the Teichmüller metric of $T(\mathbf{H})$ and let $t_{n} \rightarrow t$ as $n \rightarrow \infty$. We need to show that $H\left(t_{n},\left[h_{n}\right]\right) \rightarrow H(t,[h])$ as $n \rightarrow \infty$.

We give a lemma which is used in the proof. Let $\mathcal{G}=\hat{\mathbf{R}} \times \hat{\mathbf{R}} \backslash$ diag be the space of (oriented) geodesics in $\mathbf{H}$. Let $Q_{*}=[a, b] \times[c, d] \subset \mathcal{G}$, for $a, b, c, d$ in counterclockwise order on $\hat{\mathbf{R}}$, be a fixed "box" of geodesics such that the Liouville measure $L\left(Q_{*}\right)$ of the box $Q_{*}$ is 1 , namely,

$$
L\left(Q_{*}\right)=\log \frac{(c-a)(d-b)}{(d-a)(c-b)}=1 .
$$

Lemma 4.1. Let $\left[h_{n}\right] \rightarrow[h]$ in $T(\mathbf{H}), \mu_{n}=\mathcal{E} \mathcal{M}\left(\left[h_{n}\right]\right)$ and $\mu=\mathcal{E} \mathcal{M}([h])$. Then $\mu_{n} \rightarrow \mu$ in the following sense:

Let $Q_{*}=[a, b] \times[c, d]$ be a fixed box with $L\left(Q_{*}\right)=1$. If $Q$ is any box with $L(Q)=1$, then there exists $\gamma_{Q} \in P S L_{2}(\mathbf{R})$ such that $\gamma_{Q}(Q)=Q_{*}$. For any continuous function $\varphi: \mathcal{G} \rightarrow \mathbf{R}$ whose support is in the fixed box $Q_{*}$, we have that

$$
\mathcal{S}_{\varphi}\left(\mu_{n}, \mu\right)=\sup _{L(Q)=1}\left|\iint_{Q_{*}} \varphi d\left(\gamma_{Q}\right)_{*}\left(\mu_{n}\right)-\iint_{Q_{*}} \varphi d\left(\gamma_{Q}\right)_{*}(\mu)\right| \rightarrow 0
$$

as $n \rightarrow \infty$, where the supremum is over all boxes $Q$ with $L(Q)=1$.

Proof. Assume on the contrary that $\mu_{n} \nrightarrow \mu$ as $n \rightarrow \infty$. Then there exist a continuous function $\varphi: \mathcal{G} \rightarrow \mathbf{R}$ whose support is in $Q_{*}$ and a sequence $Q_{n_{k}}$ with $L\left(Q_{n_{k}}\right)=1$ such that

$$
\left|\iint_{Q_{*}} \varphi d\left(\gamma_{Q_{n_{k}}}\right)_{*}\left(\mu_{n}\right)-\iint_{Q_{*}} \varphi d\left(\gamma_{Q_{n_{k}}}\right)_{*}(\mu)\right| \geq \epsilon_{0}>0 .
$$

We write $n$ instead of $n_{k}$ for simplicity. Let $\nu_{n}=\left(\gamma_{Q_{n}}\right)_{*}\left(\mu_{n}\right)$ and $\zeta_{n}=\left(\gamma_{Q_{n}}\right)_{*}(\mu)$ be the push-forwards by $\gamma_{Q_{n}}$ of $\mu_{n}$ and $\mu$, respectively. By the above, at least one of the measures $\nu_{n}$ and $\zeta_{n}$ are not zero on $Q_{*}$. Let $t_{\nu_{n}}$ be a geodesic in $Q_{*}$ in the support of $\nu_{n}$ or in the interior of a stratum of $\nu_{n}$. Define $t_{\zeta_{n}}$ similarly.

Let $\delta_{n}, \delta_{n}^{\prime} \in P S L_{2}(\mathbf{R})$ be such that $\left.E^{\nu_{n}}\right|_{t_{\nu_{n}}}=\left.\delta_{n} \circ E^{\mu_{n}} \circ \gamma_{Q_{n}}^{-1}\right|_{t_{\nu_{n}}}=i d$ and that $\left.E^{\zeta_{n}}\right|_{t_{\zeta_{n}}}=\left.\delta_{n}^{\prime} \circ E^{\mu} \circ \gamma_{Q_{n}}^{-1}\right|_{t_{\zeta_{n}}}=i d$. Note that $\left\|\nu_{n}\right\|$ and $\left\|\zeta_{n}\right\|$ are bounded uniformly in $n$ (because $\left[h_{n}\right] \rightarrow[h]$ in the Teichmüller metric). It follows that there exist subsequences of $\left.E^{\nu_{n}}\right|_{\hat{\mathbf{R}}}$ and $\left.E^{\zeta_{n}}\right|_{\hat{\mathbf{R}}}$ which converge weakly on $\hat{R}$ to homeomorphisms $f$ and $g$, respectively (see [10]). Moreover, there are subsequences of $\nu_{n}$ and $\zeta_{n}$ which converge in the weak ${ }^{*}$ sense to bounded measured laminations $\nu_{*}$ and $\zeta_{*}$, respectively. We can assume without loss of generality that both sequences converge. It is clear that $\nu_{*} \neq \zeta_{*}$ because $\nu_{n}$ and $\zeta_{n}$ differ on $Q_{*}$ by a definite amount (according to the assumption). This implies that $g \circ f^{-1}$ is not in $P S L_{2}(\mathbf{R})$.

Let $\tilde{F}_{n}$ and $\tilde{G}_{n}$ be the Douady-Earle extensions of $\left.E^{\nu_{n}}\right|_{\hat{\mathbf{R}}}$ and $\left.E^{\zeta_{n}}\right|_{\hat{\mathbf{R}}}$, respectively. Let $\tilde{F}$ and $\tilde{G}$ be the Douady-Earle extensions of $f$ and $g$, respectively. Then

$$
\left|\operatorname{Belt}\left(\tilde{F}_{n}\right)-\operatorname{Belt}\left(\tilde{G}_{n}\right)\right| \rightarrow 0
$$

uniformly on compact subsets of $\mathbf{H}$ because $\left.E^{\nu_{n}}\right|_{\hat{\mathbf{R}}}=\delta_{n} \circ h_{n} \circ \gamma_{Q_{n}}^{-1}$ and $\left.E^{\zeta_{n}}\right|_{\hat{\mathbf{R}}}=$ $\delta_{n}^{\prime} \circ h \circ \gamma_{Q_{n}}^{-1},\left[h_{n}\right] \rightarrow[h]$ and by the conformal naturality of the Douady-Earle extension. This implies that $\operatorname{Belt}(\tilde{G})=\operatorname{Belt}(\tilde{F})$, which is a contradiction with $g \circ f^{-1} \notin P S L_{2}(\mathbf{R})$. 
We continue with the proof of the theorem. Let $d\left(\left[h_{1}\right],\left[h_{2}\right]\right)$ denote the Teichmüller distance between $\left[h_{1}\right],\left[h_{2}\right] \in T(\mathbf{H})$. Let $F_{n}$ and $G$ be the Douady-Earle extensions of $\left.E^{\left(1-t_{n}\right) \mu_{n}}\right|_{\hat{\mathbf{R}}}$ and $\left.E^{(1-t) \mu}\right|_{\hat{\mathbf{R}}}$, respectively. Assume on the contrary that $d\left(\left.E^{\left(1-t_{n}\right) \mu_{n}}\right|_{\hat{\mathbf{R}}},\left.E^{(1-t) \mu}\right|_{\hat{\mathbf{R}}}\right) \nrightarrow 0$ as $n \rightarrow \infty$. This is equivalent to the statement that there exists $z_{n} \in \mathbf{H}$ such that

$$
\left|\operatorname{Belt}\left(F_{n}\right)\left(z_{n}\right)-\operatorname{Belt}(G)\left(z_{n}\right)\right| \geq \epsilon_{0}>0 .
$$

Let $\gamma_{n} \in P S L_{2}(\mathbf{R})$ be such that $\gamma_{n}\left(z_{n}\right)=i \in \mathbf{H}$. There exist subsequences of $\left(\gamma_{n}\right)_{*}\left(\left(1-t_{n}\right) \mu_{n}\right)$ and of $\left(\gamma_{n}\right)_{*}((1-t) \mu)$ which converge in the weak ${ }^{*}$ sense. We can assume without loss of generality that both sequences converge in the weak* sense and the above lemma implies that their limits are equal. Thus there exist

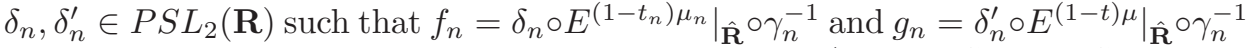
pointwise converge to the same homeomorphism of $\hat{\mathbf{R}}$. Let $\tilde{F}_{n}$ and $\tilde{G}_{n}$ be the Douady-Earle extensions of $f_{n}$ and $g_{n}$, respectively. Consequently $\operatorname{Belt}\left(\tilde{F}_{n}\right)$ and $\operatorname{Belt}\left(\tilde{G}_{n}\right)$ are close on compact subsets of $\mathbf{H}$ for $n$ large. This contradicts (1) by the conformal naturality of the Douady-Earle extension and the fact that $\gamma_{n}\left(z_{n}\right)=$ $i \in \mathbf{H}$ (see the proof of the above lemma for similar reasoning).

The above theorem gives a new proof that the Teichmüller space of an arbitrary Riemann surface is contractible. This was originally proved by Douady and Earle [1] (see [3] for contractibility of the subspace of asymptotically conformal classes).

Corollary 4.1. The Teichmüller space $T(\mathbf{H} / G)$ of a hyperbolic surface $\mathbf{H} / G$ is contractible. If $\mathbf{H} / G$ is geometrically infinite, then the subspace of $T(\mathbf{H} / G)$ consisting of all asymptotically conformal classes is also contractible.

Remark. The above theorem implies contractibility of any subspace of $T(\mathbf{H})$ as long as it is closed under earthquake paths.

\section{Minimal Rate of DeCREase toward THE BOUNDARY}

We establish a sufficient condition for a measured lamination $\mu$ on the hyperbolic plane to be trivial in terms of its rate of decrease when approaching the boundary. It is convenient to work with the unit disk $\mathbf{D}$ model of the hyperbolic plane, where the hyperbolic metric is $\rho(z)=\frac{2|d z|}{1-|z|^{2}}$. Let $I$ be a closed hyperbolic arc of length 1 in D. Denote by $\delta(I)$ the minimal Euclidean distance from $I$ to the unit circle $S^{1}=\partial \mathbf{D}$. We say that $\mu$ decreases of the order $o(\delta)$ and write $\mu=o(\delta)$ if

$$
\mu(I) / \delta(I) \rightarrow 0
$$

as $\delta(I) \rightarrow 0$ uniformly in $I$.

Proposition 5.1. Let $\mu$ be a measured lamination on $\mathbf{D}$. If $\mu=o(\delta)$, then $\mu$ is trivial, i.e. $\mu=0$.

Proof. Consider the Euclidean circle of radius $1-1 / n$ with center 0 . The hyperbolic length of the circle is $\frac{4 \pi(n-1)}{2-1 / n}$. The circle is at a Euclidean distance $1 / n$ from $S^{1}$. The assumption on $\mu$ implies that the total $\mu$-measure of the circle is $o(1 / n)$. $\frac{4 \pi(n-1)}{2-1 / n} \rightarrow 0$ as $n \rightarrow \infty$. Since each compact subset of the support of $\mu$ intersects all such circles for $n$ large enough, it follows that the $\mu$-measure of each compact set in its support is zero. 


\section{REFERENCES}

[1] A. Douady and C. J. Earle, Conformally natural extension of homeomorphisms of the circle, Acta Math. 157 (1986), no. 1-2, 23-48. MR857678 (87j:30041)

[2] C. Earle, F. Gardiner and N. Lakic, Asymptotic Teichmüller space. II. The metric structure, In the tradition of Ahlfors and Bers, III, 187-219, Contemp. Math., 355, Amer. Math. Soc., Providence, RI, 2004. MR2145063 (2006g:30078)

[3] C. Earle, V. Markovic and D. Sarić, Barycentric extension and the Bers embedding for asymptotic Teichmüller space, Complex manifolds and hyperbolic geometry (Guanajuato, 2001), 87-105, Contemp. Math., 311, Amer. Math. Soc., Providence, RI, 2002. MR1940165 (2003i:30072)

[4] D. Epstein, A. Marden and V. Markovic, Quasiconformal homeomorphisms and the convex hull boundary, Ann. of Math. (2) 159 (2004), no. 1, 305-336. MR2052356 (2005d:30067)

[5] F. Gardiner, J. Hu and N. Lakic, Earthquake curves, Complex manifolds and hyperbolic geometry (Guanajuato, 2001), 141-195, Contemp. Math., 311, Amer. Math. Soc., Providence, RI, 2002. MR1940169 (2003i:37033)

[6] F. Gardiner and D. Sullivan, Symmetric structures on a closed curve, Amer. J. Math. 114 (1992), no. 4, 683-736. MR 1175689 (95h:30020)

[7] J. Hu, Earthquake measure and cross-ratio distortion, In the tradition of Ahlfors and Bers, III, 285-308, Contemp. Math., 355, Amer. Math. Soc., Providence, RI, 2004. MR2145070 (2006m:37056)

[8] S. Kerckhoff, The Nielsen realization problem, Ann. of Math. (2) 117 (1983), no. 2, 235-265. MR690845 (85e:32029)

[9] D. Sarić, Real and complex earthquakes, Trans. Amer. Math. Soc. 358 (2006), no. 1, 233-249. MR2171231 (2006i:30063)

[10] D. Sarić, Bounded earthquakes, Proc. Amer. Math. Soc. 136 (2008), no. 3, 889-897 (electronic). MR 2361861 (2009f:30101)

[11] W. Thurston, Earthquakes in two-dimensional hyperbolic geometry, Low-dimensional topology and Kleinian groups (Coventry/Durham, 1984), 91-112, London Math. Soc. Lecture Note Ser., 112, Cambridge Univ. Press, Cambridge, 1986. MR903860 (88m:57015)

[12] W. Thurston, Three-dimensional geometry and topology, Vol. 1. Edited by Silvio Levy. Princeton Mathematical Series, 35. Princeton University Press, Princeton, NJ, 1997. MR 1435975 (97m:57016)

Department of Mathematics, Queens College of The City University of New York, 65-30 Kissena Boulevard, Flushing, New York 11367

E-mail address: Dragomir.Saric@qc.cuny.edu 